\title{
The Dynamic Scheduling of Spray Booth Machine in 4S Auto Maintenance Shop
}

\author{
Jinqi $\mathrm{Li}^{1,} \mathrm{a}^{*}$, Xiangyang $\mathrm{Li}^{1}$ and Qin $\mathrm{Yang}^{2}$ \\ ${ }^{1}$ School of management, Harbin Institute of Technology, Harbin 150001, P.R. China \\ ${ }^{2}$ School of Business, Sichuan Normal University, Chengdu 610101, P.R. China
}

ajinqili@aliyun.com

\begin{abstract}
Keywords: Bottleneck, Spray booth machine, Triplet method, Dispatching rules, Particle swarm optimization algorithm
\end{abstract}

\begin{abstract}
Theory of constraint point out that the bottleneck is a major factor which restricts the system going toward the target. Therefore, the bottleneck is the key of scheduling process in entire system. In fact, due to the factors of long processing time, high cost and few amounts, spray booth machine become the bottleneck in the whole $4 \mathrm{~S}$ auto maintenance shop. Through reasonable scheduling of spray booth machine, we can improve system's efficiency and customer's satisfaction First, combining with the real situation, we apply a triplet method to describe the spray booth scheduling problem in $4 \mathrm{~S}$ maintenance shop. On this basis, a multi-objectives model with multi-constraint is constructed for the scheduling problem. Combining with the real situation, the scheduling model also can be regard as machine in parallel with different speed and machine eligibility restriction and job's characteristic. And then, we design a two-phased heuristic algorithm based on the dispatching rules and particle swarm optimization algorithm to solve it. Finally, we show the experimental results and compares with usual methods to demonstrate the superior performance of the proposed method.
\end{abstract}

\section{Introduction}

$4 \mathrm{~S}$ auto maintenance shop includes a series of maintenance centers, such as electromechanical maintenance, sheet metal, spray painting, car beauty, and so on. It is a complex maintenance system that may serve for so many customers simultaneously for regular vehicle maintenance or preventative maintenance. As the mainly spray painting device, spray booth machine is so expensive, the processing time in this device is so long, and all these factors contribute it to become the bottleneck resources in the whole $4 \mathrm{~S}$ auto maintenance shop. Theory of constraint (TOC) has been proposed by Goldratt and Cox who illustrate that bottleneck is a major factor which restricts the system going toward the target and governs the performance of the whole system [1]. So in order to improve the performance of the overall system, the key of scheduling is bottleneck resources. Through the reasonable scheduling of spray booth machine can not only improve the performance of whole $4 \mathrm{~S}$ auto maintenance system effectively, but also reduce customers waiting time so that to promote customers satisfaction. However, scheduling in practice application areas is often not as good as expected, especially the application of simple scheduling methods such as first come first service (FCFS), shortest processing rule (SPT), and so on [2]. More effective scheduling method is needed to solve the scheduling problem.

In spray painting center, there mainly contains some spray booth machine to finish a lot of spray painting job, however, due to the difference in updating time, job requirement, cost and other factors, different spray booth machine may have different processing speed and application area. Moreover, compared with the traditional manufacturing system, 4S auto maintenance shop contact with customer's requirements more directly and lack the buffer of middleman. So it is difficult to make preparations and keep inventory for maintenance jobs and the scheduling processing is more dynamic and lack the flexibility. Therefore, the spray booth scheduling problem also can be defined as a non-identical parallel machines scheduling problem with machine eligibility and constraints of 
job's characteristics.

The computational process of parallel machine scheduling problem is very complexity and with the increase scale of this scheduling problem, the computational complexity increase intensively, the exact methods may not get the optimal scheduling solution in a short time. Therefore, many scholars mainly focus on the heuristic algorithm to solve the scheduling problem. Nérona et al. proposed a search tree based approach to solve the parallel machine scheduling problem with the constraint of release date and tails [2]. Li and Yang solved the parallel machine problem with minimizing the total weighted completion times by three types of search algorithms [3]. Torabi et al. used particle swarm optimization algorithm for a parallel machines scheduling problem with a fuzzy multi-objective [4]. On the basis of this, Ali and Behrooz proposed a discrete particle swarm optimization algorithm to solve the problem [5]. Alcan et al. considered the fuzzy processing times and applied the genetic algorithm to solve the non-identical parallel machine [6]. Balin proposed a new genetic algorithm revised from an optimum criterion and new crossover operator, and then verify the efficiency of proposed algorithm by simulating result [7]. Moreover, some other algorithms also respect to solve the parallel machine scheduling problem [8-11].

Fore-mentioned literatures have conducted a meaningful exploration for the parallel machine scheduling problem. But they mainly simplified the scheduling problem or consider a static scheduling environment. However, in practice, the scheduling of spray booth in $4 \mathrm{~S}$ auto maintenance shop contains so many kinds of dynamic events such as the uncertain processing time, stochastic job arrivals, and unexpected break downs. Moreover, the Particle swarm optimization algorithm owns the advantages of simple principle and easy implementation and it has good performance in solving dynamic scheduling problem. However, the application and extensive research of the method in the field is still inadequate. According to this, we consider the spray booth scheduling problem with multi-objective and multi-constraints and build a corresponding scheduling model. On the basis of this, we adopt the hybrid rescheduling rules to realize the dynamic scheduling process and design a two-phase heuristic method based on the hybrid dispatching rules and the encoding of particle swarm algorithm to solve the problem.

The remainder of this paper is organized as follows: In section 2 , we apply a triplet $\alpha / \beta / \gamma$ method to analyze and describe the real situation of the spray booth scheduling problem in $4 \mathrm{~S}$ auto maintenance shop. And then, a multi-objectives scheduling model with multi-constraint is designed. Section 3 present a two-phased heuristic algorithm based on the dispatching rules and the encoding of particle swarm algorithm to solve the spray booth scheduling problem. Also, combining with characteristics of the scheduling problem, we design the hybrid rescheduling rules to realize the dynamic scheduling process. In section 4 , we shows the experimental results and compares with some common methods to certify the superior performance of the proposed method. Finally, we conclude this paper by discussing the improvements achieved and extensive research in the future.

\section{Problem Description and Mathematical Model}

Problem Description. In this paper, we adopt the triplet $\alpha / \beta / \gamma$ method to describe the spray booth scheduling problem in $4 \mathrm{~S}$ auto maintenance shop. In the description, the superscript $i$ denotes any one of spray booth machine and $m$ denotes the total number of spray maintenance job, $i \in[1, m]$; the subscript $j, j \in[1, n]$ denotes any one of spray maintenance job and $n$ denotes the total number of spray maintenance job.

Resource environment. $4 \mathrm{~S}$ auto maintenance shop contains a spray booth machine set to finish a lot of spray painting job. However, these spray booth machine may have different processing speed and eligibility restriction because of the difference in the update time and specification of machine, and other factors. Generally speaking, spray booth scheduling problem can be solved in non-identical parallel machine scheduling environment.

Processing characteristics and constraints. In $4 \mathrm{~S}$ auto maintenance shop, the maintenance jobs possess the following constraints and restriction: time window constraint (include the due date $d_{j}$ and release date $r_{j}$ ), processing time $p_{j}^{i}$, machine eligibility restriction $M_{j}$, setup time $S_{j k}^{i}$, 
specifically as follows: Due date $d_{j}$ represents the expected completion time of job $j . r_{j}$ denotes the earliest time of job $j$ can start its processing procedure. The processing time of job $j$ on machine $i$ is defined as $p_{j}^{i}$. The $M_{j}$ means not all of spray booth machine are capable to process job $j$, which is determined by machine utilized restriction and jobs property. The $S_{j k}^{i}$ represents the interval between the completion time of job $j$ and starting time of job $k$ on machine $i$.

The scheduling objective. The scheduling problem of spray booth in $4 \mathrm{~S}$ auto maintenance shop is related to the benefits of customers and $4 \mathrm{~S}$ auto operator. In this paper, we attempt to optimize the interests between the two sides. Focus on customers, the scheduling target need to ensure the due date of $d_{j}$ as far as possible and minimize the tardiness time of maintenance job. Also, the tardiness can be denoted as $T_{j}=\max \left(C_{j}^{i}-d_{j}\right)$. In the equation, the $C_{j}^{i}$ represents the actual completion of maintenance job $j$. In fact, the more effective tardiness may have to consider the different weight $\omega_{j}$ of each maintenance job. For instance, when the very important client and the ordinary client enter the service system and ask for maintenance service at the same time, generally, the very important client may have higher weight of job and receive the maintenance service priority. So in this paper, we set the target of minimize the total weighted tardiness time $\omega_{j} T_{j}$ to ensure customers interests in terms of time. For $4 \mathrm{~S}$ auto operators, spray booth machine is bottleneck resources in maintenance system and improve its utilization is one of the key scheduling objective. The makespan $C_{\max }$ represents the completion time of last job to leave the maintenance system [12], namely, $C_{\max }=\max \left(C_{1}, C_{2}, \cdots, C_{n}\right)$. Minimizing the makespan can ensure the spray booth machine is fully used. To sum up, the scheduling objective in this paper is defined as $\operatorname{Min}\left[\theta_{1} \sum_{j=1}^{n} \omega_{j} T_{j}+\theta_{2} C_{\text {max }}\right]$, and $\theta_{1}, \theta_{2}$ are two parameters to eliminate the dimension. We set $\theta_{1}=1 /\left[\min \sum_{j=1}^{n} \omega_{j} T_{j}\left(X_{h}\right)\right], \theta_{2}=1 /\left[\min \left[C_{\max }\left(X_{h}\right)\right] . X_{h}\right.$ denotes the scheduling plan $h$ and more details is given in section 4.1 .

The Designing of Scheduling Model. The mathematical model is shown as follows.

$$
\begin{aligned}
& \operatorname{Min}\left[\theta_{1} \sum_{j=1}^{n} \omega_{j} T_{j}+\theta_{2} C_{\max }\right] \\
& \text { s.t. } T_{j}=\max \left(C_{j}^{i}-d_{j}\right) \quad / i \in m, j \in n / \\
& C_{j}^{i}-p_{j}^{i} \geq r_{j} \quad / i \in n, j \in n / \\
& p_{j}^{i}=Q_{j} / u^{i} \quad / i \in n, j \in n / \\
& C_{j}^{i}=\left(C_{k}^{i}+s_{j k}^{i}+p_{j}^{i}\right) \cdot x_{(t) j}^{i} / i, k \rightarrow i, j / \\
& x_{(t) j}^{i} \in\{0,1\} \quad / i \in n, j \in n, t \in C_{\max } / \\
& \sum_{t=0}^{C_{\max }} \sum_{i=1}^{m} x_{(t) j}^{i}=1 \quad / j \in n / \\
& \sum_{t=0}^{C_{\max }} \sum_{i=1}^{m} \sum_{j=1}^{n} x_{(t) j}^{i}=n \\
& d_{j} \geq 0, r_{j} \geq 0, \omega_{j} \geq 0 \quad / j \in n /
\end{aligned}
$$

Eq. (1) shows the scheduling objective in this paper. All the constraints are from Eq. (2) to Eq. (9). Constraint (2) represents the tardiness time of job $j$. Constraint (3) make sure that the operating process of job $j$ on machine $i$ can be completed. Constraint (4) measure the processing time of job $j$ on machine $i$. The $u^{i}$ represents the processing speed of machine $i$. The $Q_{j}$ represents the amounts of job $j$, which depends on the actual job property. For instance, different damage area and location of scratched vehicles may have different value of $Q_{j}$. Constraint (5) measure the actual completion time of job $j$ on machine $i$. And $i, k \rightarrow i, j$ denotes the processing order, namely the machine $i$ is used for job $k$ before used for job $j$. Constraint (6) indicates whether the job $j$ is processed on machine $i$ at time $t$, and it can divided into two cases. Case 1: when the job $j$ is processed on machine $i$ at time $t$, the value of $x_{(t) j}^{i}$ is 1. Case 2: other situation, the value is 
0. Constraint (7) make sure that the maintenance job only use the machine one time in the scheduling process. Constraint (8) ensures all the maintenance jobs can be completed. Constraint (9) define the value range of due date, release date and weight of job $j$.

\section{The Design of Scheduling Method}

Firstly, we adopt the hybrid rescheduling rules to realize the dynamic scheduling process of spray booth machine in $4 \mathrm{~S}$ auto maintenance shop. And then, we design a two-phased heuristic algorithm based on hybrid dispatching rules and particle swarm optimization algorithm to solve the scheduling problem.

The Designing of Rescheduling Rules. The dynamic rescheduling rules mainly involves three aspects: event-driven rescheduling, cyclic rescheduling, and hybrid rescheduling. Hybrid rescheduling rules has advantages both in event-driven rescheduling and cyclic rescheduling and it can respond to actual dynamic environment preferably and steadily. Focusing on the practical problem, this study attempt to adopt the job-based hybrid rescheduling rules to realize the dynamic scheduling process. The hybrid rescheduling window is shown in Fig. 1.

Case 1: In general, rescheduling periodically. Each time $e$ maintenance jobs are carried out in the local optimal schedule, and we record the moment of completing $e$ maintenance jobs as the rescheduling moment.

Case 2: when the specific events happen, if only $e^{\prime}\left(e^{\prime}<e\right)$ maintenance jobs have been completed at this time, rescheduling is also needed. In the rolling windows, there are at moste maintenance jobs and it less than the number of the maintenance jobs in the predicted windows, namely $L(\mathrm{~g})>e$. These specific events mainly include the abrupt and urgent jobs, the variation of the priority of the jobs, machine breakdowns etc.

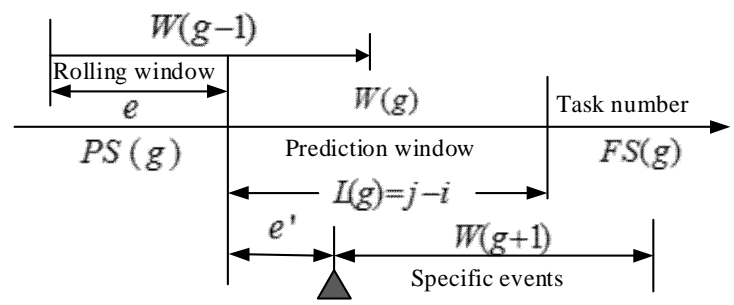

Fig. 1 Hybrid rescheduling strategy based on jobs.

Phase One: Seeking of Initial Solution by the Hybrid Dispatching Rules. The traditional heuristic algorithms generate the initial solution randomly based on certain pattern and it may have impact on convergence process and performance of algorithm. Application of some simple rules in the process of seeking the initial solution may perform better. In this paper, we composite the least flexible rules and apparent tardiness cost with setups rules to realize the primary solving in each rescheduling process and regard it as the initial solution for iterative optimization in next phase by the encoding of particle swarm optimization algorithm.

The allocation of maintenance jobs. According to the least flexible machine rule (LFM) and least flexible job rule (LFJ) [12], we can assign all maintenance jobs in prediction window to each spray booth machine. Namely, we allocate the spray booth machine which has the minimum $M_{j}$ to the least flexible maintenance job. If the different maintenance jobs have equal $M_{j}$, allocate it randomly.

Determining the processing order. The ultimate objective of scheduling is allocate all jobs to each machine reasonably. Sometimes one machine may corresponding to a maintenance job set and there exist the processing order on each machine. In this paper, we use the apparent tardiness cost with setups rule (ATCS) to realize the primary schedule of job's processing order on each spray booth machine. Each time we need calculate the job index by the formula (10) and choose the job who owns the larger job index to execute the primary schedule. 


$$
I_{j}^{i}(t, k)=\frac{w_{j}}{p_{j}^{i}} \cdot \exp \left[-\frac{\max \left(d_{j}-p_{j}^{i}-t, 0\right)}{K_{1} \bar{p}}\right] \cdot \exp \left(-\frac{s_{j k}^{i}}{K_{2} s}\right)
$$

In the equation, $\bar{p}$ denotes the average processing time of the rest unscheduled jobs. $K_{1}$ and $K_{2}$ denotes the ratio parameter which related to the due date and setup time, respectively. $\bar{s}$ denotes the average setup time.

Phase Two: Iterating by Encoding of Particle Swarm Optimization Algorithm. Particle Swarm Optimization algorithm (PSO) first proposed by Kennedy and Eberhart [13], which is an evolutionary computation method based on swarm intelligence. Due to the advantages of simple principle and easy implementation, PSO is widely used in the research about engineering technology and manufacturing system. In this paper, we encode the particle's position, velocity and update rules based on the actual situation of spray booth scheduling problem to iterate and get the optimal solution finally.

The definition of each particle. In the particle swarm, each particle has been set as a two dimensional vector, which denote the processing order of maintenance job on each spray booth machine. Each particle can be regard as a scheduling plan.

$$
X_{h}=\left[\begin{array}{l}
A_{h} \\
B_{h}
\end{array}\right]=\left[\begin{array}{l}
a_{h 1}, \cdots, a_{h j}, \cdots, a_{h n} \\
b_{h 1}, \cdots, b_{h j}, \cdots, b_{h n}
\end{array}\right]
$$

$A_{h}=\left[a_{h 1}, \cdots, a_{h j}, \cdots, a_{h n}\right]$ denotes the machine vector of particle $h$ (also called plan $h$ ) and $a_{h j}$ determines which spray booth machine is used for maintenance job $j$ in plan $h$. In this paper, we define $a_{h j}$ is an integer number and $a_{h j} \in[1, m]$. The processing order vector $B_{h}=\left[b_{h 1}, \cdots, b_{h j}, \cdots, b_{h n}\right]$ is defined as a random vector and the smaller the value of $b_{h j}$, the priority processing on corresponding machine. For each initial particle, we define $b_{h j} \in[0,2]$. For example: $X_{h}=\left[\begin{array}{l}A_{h} \\ B_{h}\end{array}\right]=\left[\begin{array}{c}1,2 \\ 0.49,1.71,1.58,0.69,0.35,0.93\end{array}\right]$. In this case, 6 maintenance job are processed in 3 spray booth machine. According to the value of $b_{h j}$, we can know the processing order on machine 1 is from job 1 to job 4 . On machine 2, the processing order is from job 5 to job 2 . On machine 3, the processing order is from job 6 to job 3.

Choosing the optimal particle. Combining with actual situation of scheduling problem, we encoding the PSO to get the optimal solution with iterative computation. In each iteration, each particle update themselves by tracking two optimal particle, namely local optimal particle and global optimal particle. In this paper, the scheduling objective is $\operatorname{Min}\left[\theta_{1} \sum_{j=1}^{n} \omega_{j} T_{j}\left(X_{h}\right)+\right.$ $\theta_{2} C_{\max }\left(X_{h}\right)$ ], which can be used to evaluate the performance of each particle. In this paper, we use objective function value of each particle to mark and choose the local optimal particle $X_{h p}$ and global optimal particle $X_{g}$. Case 1: For particle $h$, if the optimal solution of a particle has found in itself sub-queue, recorded the particle as local optimal particle $X_{h p}$. Case 2: If the optimal solution of a particle has found in entire particle swarm, recorded the particle as the global optimal particle $X_{g}$.

The definition of particle's velocity and position. In fact, the iterate process of PSO also can be regard as each particle "fly" to optimal particle by certain velocity and rules. Combing with the actual situation of scheduling problem, we just define the velocity for processing order vector of each particle. For particle $h$, the velocity of processing order vector is defined as $V B_{h}=$ $v b_{h 1}, v b_{h 2}, \cdots, v b_{h j}, \cdots, v b_{h n} . v b_{h j}$ is a random number.

The updating of particle's velocity and position. In each particle $h$, the update rule of machine vector and processing order vector is shown as follows: Step 1: We adopt the cross-iteration method to update the machine vector. Namely, for updating particle $h$, first we choose two machine parameter from machine vector $A_{h}$ randomly. And then choosing two machine parameter from local optimal particle $X_{h p}$ and global optimal particle $X_{g}$ randomly. Finally, we exchange the value of machine parameter chosen from particle $h$ with the value of machine parameter chosen 
from local optimal particle $X_{h p}$ and global optimal particle $X_{g}$, respectively. Step 2: In this paper, we use Eq. (12) and (13) to update the processing order vector:

$$
\begin{aligned}
& V B_{h}=w \times V B_{h}+c_{1} \times \operatorname{random}() \times\left(B_{h p}-B_{h}\right)+c_{2} \times \operatorname{random}() \times\left(B_{g}-B_{h}\right) \\
& B_{h}=B_{h}+V B_{h}
\end{aligned}
$$

In Eq. (12), random () denotes the random number and it belongs to $[0,1] . c_{1}, c_{2}$ are learning factor, generally, $c_{1}=c_{2}=2$. Generally speaking, the larger the value of parameter $w$, the stronger the capability of global search, otherwise, it tends to conduct the local search. According traditional method, we set the initial value of parameter $w$ is 0.9 , and then linear diminish to 0.4 with the number of iterations. In order to ensure the diversity of scheduling plan, we set the value of $v b_{h j}$ in the interval $[-2,2]$. In the process of iterations, if the value of $v b_{h j}$ exceeds the given interval, taking the upper or lower as the value of $v b_{h j}$.

The Procedure of the Two-Phase Method. Step 1: Define the number of particles, generate all the positions and velocities of particles based on phase one and two, and set the maximum number of iterations. Step 2: Generate the corresponding scheduling plans according to the particles, and calculate their objective function value according to the scheduling model. Step 3: Compare particle's objective function value and choose the local optimal solution and global optimal solution. Step 4: Update the velocity and position of the particles according to Eq. (12) and (13). Step 5: If the predefined iteration number is reached, then terminate the algorithm and output the global optimal solution. Otherwise, go back to step 2.

\section{Computational Experiment and Discussion}

Basic Setup. According to the investigation and collection of fundamental data of the $4 \mathrm{~S}$ auto maintenance shop, we generate the value range of simulation parameter as follows: The number of maintenance jobs each day in $4 \mathrm{~S}$ auto maintenance shop which need to use spray booth machine is in the interval $[12,20]$. The number of spray booth machine in $4 \mathrm{~S}$ auto maintenance shop is in the interval $[3,6]$. The release date $r_{j}$ obeys discrete uniform distribution in the interval $[0,6]$. For each maintenance job $j, Q_{j}$ obeys the uniform distribution in the interval $[4,16]$. For spray booth machine $i$, the setup time $s_{j k}^{i}$ obeys the uniform distribution in the interval $[0,3]$. In order to avoid the expected completion time is suitable, we set $d_{j}=r_{j}+\lambda p \cdot p$ denotes the mean processing time of all jobs on different spray booth machine and the parameter $\lambda$ obeys the uniform distribution in the interval [2,5]. In addition, we set the weight of each maintenance job obey normal distribution in the interval $[1,3]$ and consider five jobs as the rolling windows. Each computation group $I$ is marked by $n \times m\left[A\left(m_{1}\right), B\left(m_{2}\right), C\left(m_{3}\right)\right] . n$ denotes the total number of maintenance jobs, $m$ denotes the total number of spray booth machine. $A, B, C$ are used to represent three types of spray booth machine group with different processing speed. $m_{1}, m_{2}, m_{3}$ within the bracket denotes the number of the corresponding types of machine, respectively. Assume that $15 \%$ of maintenance jobs only can be processed on $C$ identical machine. Set the maximum number of iterations is 30 .

Evaluation of Algorithms. In order to evaluate the performance of proposed method, we compare its simulation result with traditional particle swarm optimization algorithm (PSO) and genetic algorithm (GA) based on different computation group. In this paper, all algorithms are achieved by MATLAB programming and we conduct the computational experiment on a PC with core i5 processor running at $2.50 \mathrm{GHz}$ of the dominant frequency. In this paper, we adopt the performance index $p(H, S)$ to evaluate these algorithm [14]. Let $T(H, I)$ denotes the objective function value achieved by approach $H$ for the given simulation instance $I$. Also, $S$ denotes a set of simulation instances with some characteristics. We define $B E S T(I)$ as the best solution obtained by any of these methods applied to instance $I$. The scheduling performance index $p(H, S)$ of each method based on given instance is calculated by following formula: 


$$
p(H, S)=\frac{\sum_{I \in S} T(H, I)}{\sum_{I \in S} B E S T(I)}
$$

Computational Results. The scheduling performance index of these methods with different simulation instance is shown in Table 1.

Table 1. The comparison of scheduling performance.

\begin{tabular}{cccc}
\hline Problems & \multicolumn{3}{c}{ Scheduling performance index } \\
\cline { 2 - 4 }$n \times m$ & Improved PSO & PSO & GA \\
\hline $12 \times 3$ & 1.000 & 1.019 & 1.021 \\
$16 \times 3$ & 1.000 & 1.027 & 1.033 \\
$16 \times 4$ & 1.000 & 1.031 & 1.037 \\
$20 \times 4$ & 1.000 & 1.042 & 1.050 \\
$20 \times 6$ & 1.000 & 1.047 & 1.051 \\
\hline
\end{tabular}

1. Under the same type of simulation problem, the scheduling performance index of proposed algorithm is 1 and superior to other scheduling methods significantly.

2. Under the same number of spray booth machine, the scheduling performance index of proposed method is also the best with the increase of job size.

3. Under the same number of maintenance job, the scheduling performance index of proposed method is also the best with the increase of amounts of spray booth machine.

4. With the increase of problem size, the performance index of other scheduling methods grow. It means the objective function value calculated by other methods is deviate from optimal objective function value gradually. However, the scheduling performance index of proposed method is always 1. That is, the proposed method always can get the optimal solution of the scheduling problem. In conclusion, the proposed method in this paper is more suitable for solving spray booth scheduling problem in $4 \mathrm{~S}$ auto maintenance shop.

\section{Summary and Conclusions}

$4 \mathrm{~S}$ auto maintenance shop contains a series of different equipment to support the different maintenance jobs (such as electromechanical test, sheet metal, spray painting, and so on) for customers. However, because of the long processing time, few amounts, and high cost of spray painting equipment, it is the bottleneck resources and become the key of scheduling in whole maintenance system. In this paper, we build a mathematical model of the scheduling problem based on the analysis of scheduling objective, machine environment, process characteristic and constraints which can be easily extended to other job shop scheduling problems. Moreover, we proposed an improve PSO to solve it and the computational tests show that the proposed method is more suitable to solve the non-identical parallel machine scheduling problem and the finally the maintenance services system's performance and customer's satisfaction has been improved.

Furthermore, some very interesting and extend research we can attempt as shown below: First, the theory of constraint points out the bottleneck restricts the performance of whole system, however, the truth is more good rules and methods are needed to identify the importance of bottleneck. Second, considering the real situation, the earliness may also affect performance of the overall system. For instance, a customer leave car to maintenance and pick up at a fixed time. When the actual completion time of job is earlier than the appointed time, it means the $4 \mathrm{~S}$ auto shop may have to pay unnecessary inventory cost. So we also need pay attention to earliness penalty in the extend research. Third, Modern manufacturing and service system not only has the feature of traditional job shop scheduling problem, but also involves customer's participation. However, Customers are bound rationality, which means customer not always require maximizing benefits. 
Based on this, we take the customer's perception into consideration of scheduling problem is more realistic.

\section{Acknowledgements}

We are grateful for the supported by National Natural Science Foundation of China (91024028); National Natural Science Foundation of China (71401118); Ministry of Education Youth Foundation of China (13YJC630202).

\section{References}

[1] E. M. Goldratt, J. Cox, D. Whitford, The goal: a process of ongoing improvement, North River Press, New York, 1984.

[2] E. Néron, F. Tercinet, F. Sourd, Search tree based approaches for parallel machine scheduling, Comput. Operat. Res. 35 (2008) 1127-1137.

[3] K. Li, S. L. Yang, Non-identical parallel-machine scheduling research with minimizing total weighted completion times: models, relaxations and algorithms, Appl. Math. Model. 33 (2009) 2145-2158.

[4] S. A. Torabi, N. Sahebjamnia, S. A. Mansouri, M. A. Bajestani, A particle swarm optimization for a fuzzy multi-objective unrelated parallel machines scheduling problem, Appl. Soft Comput. 13 (2013) 4750-4762.

[5] A. H. Kashan, B. Karimi, A discrete particle swarm optimization algorithm for scheduling parallel machines, Comput. Ind. Eng. 56 (2009) 216-223.

[6] P. Alcan, H. Ballgil, A genetic algorithm application using fuzzy processing times in non-identical parallel machine scheduling problem, Adv. Eng. Software. 45 (2012) 272-280.

[7] S. Balin, Non-identical parallel machine scheduling using genetic algorithm, Exp. Syst. Appl. 38 (2011) 6814-6821.

[8] Z. H. Jia, Y. T. Leung, A meta-heuristic to minimize makespan for parallel batch machines with arbitrary job sizes, European J. Oper. Res. 240 (2014) 649-665.

[9] D. Anghinolfi, M. Paolucci, Parallel machine total tardiness scheduling with a new hybrid meta heuristic approach, Comput. Oper. Res. 34 (2007) 3471-3490.

[10] A. Obeid, S. Dauzère-Pérès, C. Yugma, Scheduling job families on non-identical parallel machines with time constraints, Ann. Oper. Res. 213 (2011) 221-234.

[11] H, Shams, N. Salmasi, Parallel machine scheduling problem with preemptive jobs and transportation delay, Comput. Oper. Res. 50 (2014) 14-23.

[12] M. L. Pinedo. Scheduling: Theory, Algorithms, and Systems, second ed., Prentice Hall, New Jersey, 2002.

[13] J. Kennedy, R. Eberhart. Particle swarm optimization. IEEE international conference on neural networks IV, 4 (1995) 1942-1948.

[14] E. Demirkol, S. Mehta, R. Uzsoy, A computational study of shifting bottleneck procedures for shop scheduling problems, J. Heurist. 3 (1997) 111-137. 